\title{
ADSORÇÃO DE NITRATO EM SOLOS DE CERRADO DO DISTRITO FEDERAL¹
}

\author{
JULIANA RIBEIRO ALEXANDRE DE OLIVEIRA ${ }^{2}$, LOURIVAL VILELA ${ }^{3}$ e MIGUELANGELAYARZA ${ }^{4}$
}

\begin{abstract}
RESUMO - Os objetivos deste trabalho foram verificar a adsorção de nitrato e identificar as propriedades do solo que mais influenciam este fenômeno, em um Latossolo Vermelho-Escuro, com 56\% de argila, em Planaltina, DF. Em 1996 foram coletadas amostras de solo nas profundidades de 0-20, 20-40, 40-80, 80-100 cm, de um experimento com cinco anos de duração, em uma área de cerrado típico e em duas áreas cultivadas com soja e milho, onde o solo apresentava inversão de carga em profundidade. Também foram coletadas amostras em um solo sob cerradão, com carga elétrica líquida negativa em profundidade. A relação entre nitrato adsorvido e nitrato na solução foi descrita pela equação de Freundlich e as relações entre o nitrato adsorvido e algumas propriedades físico-químicas do solo foram estudadas por meio de regressões múltiplas. O nitrato adsorvido aumentou com a profundidade em todos os tratamentos. Esta adsorção foi maior nos solos sob cerrado e cerradão. $\mathrm{O} \Delta \mathrm{pH}$ não afetou a adsorção de nitrato. A correlação entre nitrato adsorvido, matéria orgânica e sulfato extraível foi negativa. A adsorção de nitrato aumentou com a redução da matéria orgânica em profundidade e com a redução de sulfato extraível.
\end{abstract}

Termos para indexação: sulfato, gesso agrícola, composto inorgânico, matéria orgânica, carga elétrica do solo.

\section{NITRATE ADSORPTION IN CERRADO OXISOILS}

\begin{abstract}
This work aimed to assess the rate of nitrate adsorption and identify the soil properties that most affect it, in a Dark-Red Latosol, with 56\% clay content, in Planaltina, DF, Brazil. Soil samples were collected from areas with four different management systems, at depths of 0-20, 20-40, 40-80 and 80-100 cm. Three of the areas were obtained from an on-going five-year experiment, i.e., native grass savannah land and two areas cultivated with soybeans and maize that received different amounts of liming, sulphate and other nutrients and whose soils had inverted charges in depth. The fourth area was a woody savannah land, with negative net electric charge in depth. The relationship between nitrate adsorbed and nitrate in solution, was described by Freundlich equation, and the relationship between nitrate adsorbed and some soil physical-chemical properties was studied by multiple regression. $\mathrm{Ni}$ trate adsorption increased with soil depth in all treatments. Nitrate adsorption was higher in the native grass and woody savannah than in the other treatments. The $\Delta \mathrm{pH}$ as an index of net electric charge did not affect nitrate adsorption. There was a negative correlation between nitrate adsorption, organic matter and extractable sulphate. Nitrate adsorption increased with organic matter reduction in the deep layers of the soil and with decrease in extractable sulphate.
\end{abstract}

Index terms: sulphate, gypsum, inorganic compounds, organic matter, soil electric charge.

\section{INTRODUÇÃO}

O processo de nitrificação em solos bem drenados da região dos Cerrados é intenso (Suhet et al., 1986),

\footnotetext{
${ }^{1}$ Aceito para publicação em 8 de julho de 1999.

${ }^{2}$ Eng. Agrôn., Bolsista PIBIC-UnB.

${ }^{3}$ Eng. Agrôn., M.Sc., Embrapa-Centro de Pesquisa Agropecuária dos Cerrados (CPAC), Caixa Postal 08223, CEP 73301-970 Planaltina, DF. E-mail: lvilela@cpac.embrapa.br

${ }^{4}$ Eng. Agrôn., Ph.D., Consultor, Embrapa-CPAC
}

o que contribui para aumentar a lixiviação de $\mathrm{N}$ para camadas do solo onde a atividade radicular é baixa, aumentando as perdas deste nutriente. Portanto, em solos onde ocorre adsorção de nitrato na superfície coloidal, espera-se maior eficiência da adubação nitrogenada pela redução das perdas por lixiviação deste íon.

Em solos com predominância de minerais de argila do tipo 2:1, as cargas negativas desses minerais repelem o íon nitrato de sua superfície, resultando em um movimento mais rápido desse ânion que da 
água do solo (Kinjo et al., 1978). Essa lixiviação de N é uma fonte potencial de perda desse nutriente do sistema solo-planta, e ao mesmo tempo pode atuar como elemento poluente de lençóis freáticos, bem como de lagos e águas superficiais (Newbould, 1989).

A adsorção de nitrato em solos ricos em óxidos de ferro e de alumínio e materiais amorfos que ocorrem na região tropical foi observada por Kinjo \& Pratt (1971). Este fenômeno teria o efeito de retardar o movimento do íon, em relação ao movimento da água (Raij \& Camargo, 1974; Kinjo et al., 1978), e assim aumentar a eficiência da adubação nitrogenada através da redução das perdas por lixiviação do nitrato (Bowen et al., 1993). A adsorção é causada pela atração eletrostática entre o nitrato e os sítios positivamente carregados destes materiais. A retenção do nitrato está relacionada às propriedades físicas e químicas destes solos, tais como pH, matéria orgânica, superfície de adsorção e concentração eletrolítica (Black \& Waring, 1979).

A carga elétrica líquida relativa de um solo pode ser estimada pela diferença $\Delta \mathrm{pH}$ entre o $\mathrm{pH}$ em KCl $1 \mathrm{~N}$ e $\mathrm{pH}$ em $\mathrm{H}_{2} \mathrm{O}$. O $\Delta \mathrm{pH}$ indica se o $\mathrm{pH}$ do solo está abaixo ou acima do ponto de carga zero (pcz). Portanto, se o $\Delta \mathrm{pH}$ for positivo, o $\mathrm{pH}$ do solo está abaixo do ponto de carga zero e os colóides apresentam carga líquida positiva. $\mathrm{O}$ inverso ocorre, se o $\Delta \mathrm{pH}$ for negativo (Parfitt, 1980). Na região dos Cerrados ocorrem solos, principalmente latossolos, que apresentam o fenômeno de inversão de carga, ou seja, o $\Delta \mathrm{pH}$ torna-se maior que zero em subsuperfície, o que indica o predomínio de carga elétrica líquida positiva. No Distrito Federal, Goiás e Triângulo Mineiro, solos que apresentam $\Delta \mathrm{pH}>0$ e $\Delta \mathrm{pH}=0$ são freqüentes (Embrapa, 1978; Departamento Nacional da Produção Mineral, 1983). A carga elétrica líquida destes solos, tomando-se como índice o $\Delta \mathrm{pH}$, pode ser explicada pelas proporções entre os teores de matéria orgânica e argilominerais silicatados e oxídicos. A matéria orgânica e a caulinita são as principais fontes de cargas negativas nos solos dos Cerrados, destacando-se a primeira (Silva \& Resck, 1997). Nas camadas inferiores, com o decréscimo de matéria orgânica, os valores de $\Delta \mathrm{pH}$ podem se aproximar de zero ou se tornar positivos (Embrapa, 1978).

Em solos que apresentam inversão de carga em subsuperfície, a retenção de nitrato deve ser maior do que naqueles em que a carga elétrica líquida é negativa em todo o perfil.

Este trabalho teve por objetivos avaliar a adsorção de nitrato e identificar as propriedades do solo que mais influenciam este fenômeno.

\section{MATERIAL E MÉTODOS}

A adsorção de nitrato foi estudada em amostras de solo coletadas, em 1996, em quatro áreas com diferentes sistemas de manejo, nas profundidades de $0-20,20-40,40-80$, 80-100 cm. Essas amostras foram compostas de 12 subamostras na primeira profundidade, e de quatro subamostras nas demais. Três das áreas estudadas foram de um experimento em andamento desde 1991, na EmbrapaCentro de Pesquisa Agropecuária dos Cerrados (CPAC), em Planaltina, DF, sendo uma de cerrado típico e duas cultivadas com soja e milho. O solo era um Latossolo Vermelho-Vermelho, com 56\% de argila, gibbsítico (Macedo $\&$ Bryant, 1987) e apresentava $\Delta \mathrm{pH}$ positivo em profundidade. As áreas cultivadas foram submetidas a diferentes tipos de fertilização, denominados de F1 e F2. As quantidades de calcário, sulfato e nutrientes aplicados nos dois níveis de fertilidade são apresentadas na Tabela 1. A quarta área foi um Latossolo Vermelho-Escuro, com 56\% de argila, caulinítico (Lins \& Cox, 1989) sob cerradão nativo, com $\Delta \mathrm{pH}$ negativo em todo o perfil. Nas amostras de solo foram analisados: $\mathrm{pH} \mathrm{em} \mathrm{H}_{2} \mathrm{O}(1: 2,5), \mathrm{pH} \mathrm{em} \mathrm{KCl}$ $1 \mathrm{~mol} / \mathrm{L}(1: 2,5)$, matéria orgânica (MO), sulfato extraível, $\mathrm{Al}, \mathrm{Ca}+\mathrm{Mg}, \mathrm{Pe} \mathrm{K}$ (Tabela 2). O sulfato foi extraído com $\mathrm{Ca}\left(\mathrm{H}_{2} \mathrm{PO}_{4}\right)_{2} 0,01 \mathrm{~mol} / \mathrm{L}$, na relação solução extratora:solo

TABELA 1. Calagem e adubação realizadas no período de cinco anos nos tratamentos F1 e F2, nas áreas cultivadas com milho e soja.

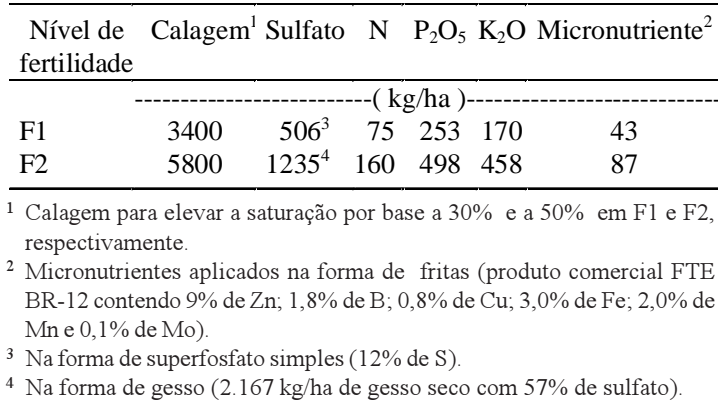


5 mL:1 g, com 24 horas de extração (Barrow, 1967). A detecção foi feita por turbidimetria com cloreto de bário. As demais determinações foram realizadas de acordo com Embrapa (1997)

As amostras de solo foram equilibradas com solução de $\mathrm{Ca}\left(\mathrm{NO}_{3}\right)_{2}$, nas concentrações de $0,20,40,80,160$ e $320 \mathrm{mg}$ de nitrato/ $\mathrm{kg}$ de solo, numa relação de $15 \mathrm{~g}$ de solo: $15 \mathrm{~mL}$ de solução; as suspensões foram agitadas por uma hora e centrifugadas por dez minutos a $10.000 \mathrm{rpm}$ (Cahn et al., 1992). A composição química do sobrenadante e da solução oclusa foi considerada equivalente (Cahn et al., 1992). O nitrato no sobrenadante foi determinado em cromatógrafo iônico Modelo IC 100, YEWYokogawa Hokushin Eletric.

O nitrato adsorvido foi estimado pela diferença entre o adicionado e o determinado na solução sobrenadante pela equação:

$\mathrm{Nx}=(\mathrm{V} \times \mathrm{Nv}+\mathrm{Ni} \times \mathrm{W}-\mathrm{Nt}(\mathrm{V}+\mathrm{Vi})) / \mathrm{W}$

onde:

$\mathrm{Nx}=$ quantidade de nitrato adsorvida por unidade de peso de solo (W) secado ao ar;

$\mathrm{Nt}$ = concentração de nitrato na solução em equilíbrio;

$\mathrm{Nv}=$ concentração de nitrato em volume $(\mathrm{V})$ de solução adicionado ao solo;

$\mathrm{Ni}=$ concentração inicial de nitrato no solo, em base seca; $\mathrm{Vi}=$ volume inicial de água no solo secado ao ar.

A relação entre nitrato adsorvido e na solução foi descrita pela equação de Freundlich: $\mathrm{Y}=\mathrm{aX}{ }^{\mathrm{b}}$ (Eq. 2), onde $\mathrm{Y}$ é o nitrato adsorvido em $\mathrm{mmol}_{\mathrm{c}} \mathrm{kg}$, $\mathrm{X}$ é o nitrato na solução em $\mathrm{mmol}_{\mathrm{c}} / \mathrm{L}$, e a e b são constantes. O ajuste dessa equação aos dados de cada profundidade foi realizado por meio de regressão não-linear do programa SAS (SAS Institute, 1989). A equação de Freundlich é a que melhor descreve os dados das concentrações adsorvidas quando a máxima adsorção não é determinada (Bohn et al., 1979; Parfitt, 1980). A relação entre nitrato adsorvido e algumas variáveis do solo foi estudada na dose mais alta de N (320 mg de nitrato/kg de solo). Selecionou-se a dose de $320 \mathrm{mg}$ de nitrato/kg de solo (144 kg de N/ha), por ser a que mais diferenciou a adsorção de nitrato no solo entre as profundidades e os sistemas estudados. Essa relação foi obtida por meio de regressões múltiplas, estimadas pelo método passo a passo do programa Statistical Analysis System (SAS Institute, 1989).

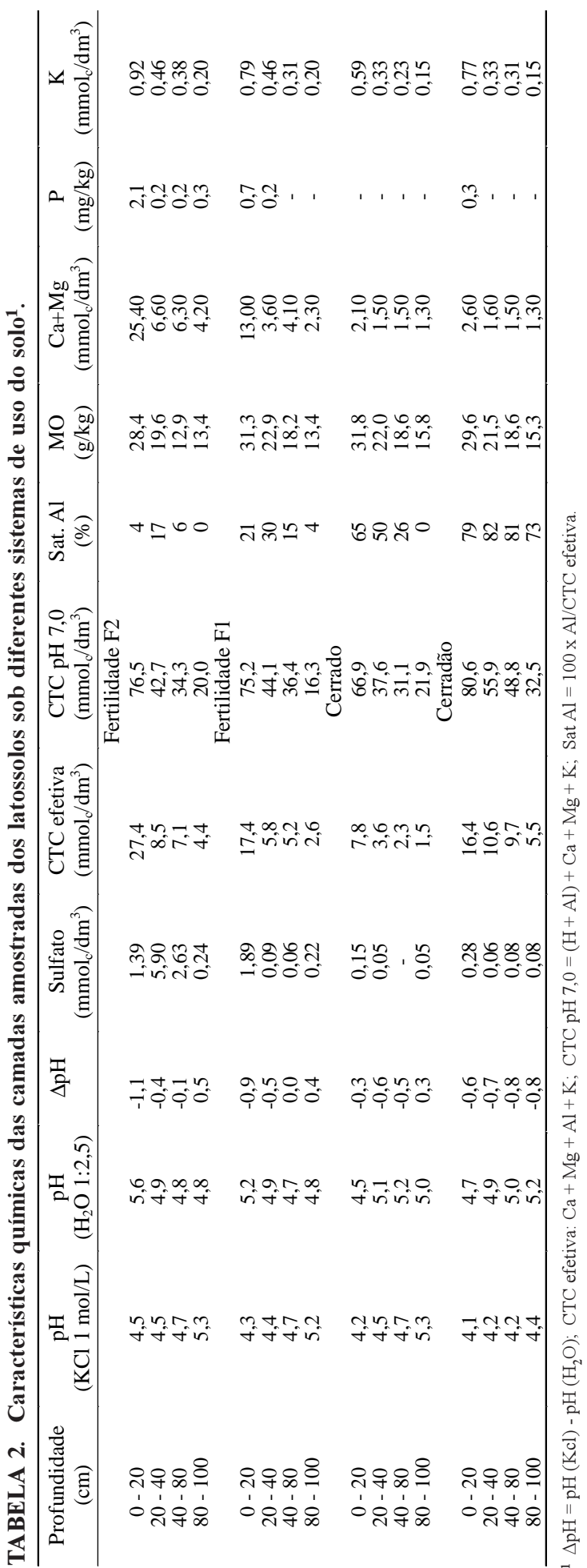

Pesq. agropec. bras., Brasília, v.35, n.6, p.1199-1205, jun. 2000 


\section{RESULTADOS E DISCUSSÃO}

A adsorção de nitrato aumentou em profundidade e foi maior nos solos sob vegetação nativa (cerrado típico e cerradão) do que nos cultivados (Fig. 1). A maior adsorção do nitrato nas camadas mais profundas também foi observada por Cahn et al. (1992) em um Latossolo Vermelho-Amarelo da Amazônia. As adsorções de nitrato na camada $80-100 \mathrm{~cm}$ do cerrado e cerradão, calculadas a partir dos parâmetros da equação de Freundlich (Tabela 3), foram, em média, $32 \%$ mais altas do que as obtidas por Cahn et al. (1992) na camada de 90-120 cm de profundidade.

$\mathrm{Na}$ camada de $0-20 \mathrm{~cm}$ de profundidade do tratamento F2, a adsorção de nitrato, além de ter sido muito pequena, apresentou uma alta variabilidade (CV>100\%). Por isso, não foi ajustada a equação de Freundlich aos dados dessa camada.

A baixa adsorção de nitrato nas camadas superficiais se deve, provavelmente, a maior quantidade de cargas negativas (Tabela 2) e competição com outros ânions. Segundo Black \& Waring (1979), a adsorção de nitrato é pouco provável em solos onde o $\Delta \mathrm{pH}$ é negativo e maior que 1,5 .

Nos sistemas cerrado e cerradão, pelos parâmetros das equações ajustadas para cada profundidade (Tabela 3), pode-se observar um decréscimo nos valores do parâmetro $b$ nas camadas inferiores, em relação à camada superior do solo. Segundo Parfitt (1980), o parâmetro b da equação de Freundlich indica afinidade (energia de ligação) por sítios de troca e decresce exponencialmente com o aumento da adsorção.

Apesar de o solo sob cerradão apresentar carga líquida negativa $(\Delta \mathrm{pH}<0)$ em todas as camadas estudadas, a adsorção de nitrato foi semelhante à do solo sob cerrado, que apresenta inversão de carga em profundidade. A afinidade (b) de adsorção de nitrato, na média das quatro profundidades foi a mesma para ambos os solos $(0,82)$. Black \& Waring (1976) verificaram uma forte correlação entre adsorção de nitrato e carga líquida estimada através do $\Delta \mathrm{pH}$. Esses autores observaram que a adsorção de nitrato

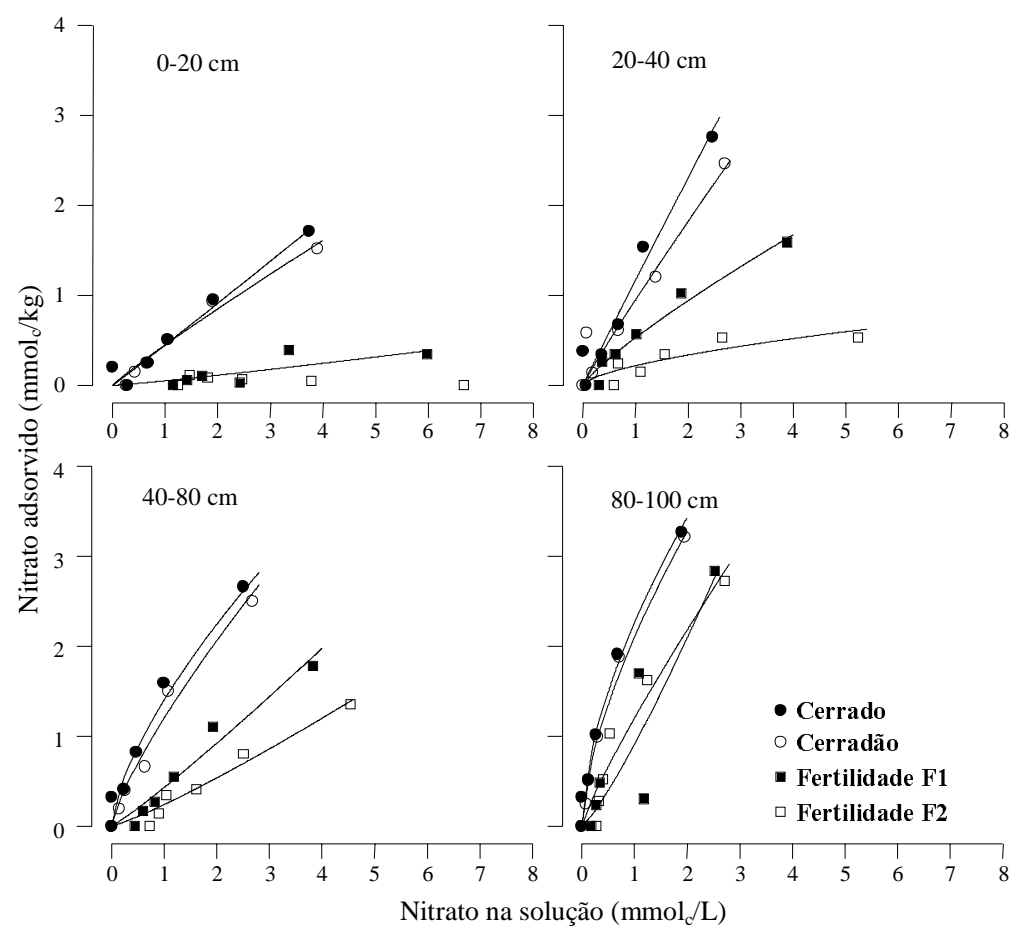

FIG. 1. Adsorção de nitrato nas amostras de solo dos quatro tratamentos nas profundidades estudadas. 
também ocorre quando a carga líquida é negativa, o que indica que as cargas positivas presentes são neutralizadas, pelo menos em parte, pelo nitrato, e não pelas cargas negativas adjacentes.

A correlação entre nitrato adsorvido e $\mathrm{pH}$ em $\mathrm{H}_{2} \mathrm{O}$, $\Delta \mathrm{pH}, \mathrm{MO}$ e sulfato extraível foi significativa apenas para as duas últimas variáveis. A equação de regressão múltipla relacionando nitrato adsorvido com os teores de MO e sulfato extraível indica que a adsorção de nitrato diminuiu com o aumento dos teores de matéria orgânica e sulfato extraível (Fig. 2). Observa-se, na Fig. 1, que as diferenças na adsorção de nitrato entre os tratamentos F1 e F2 foram maiores nas camadas de 20-40 e 40-80 cm de profundidade. As quantidades de sulfato extraível nessas duas camadas do tratamento F2 foram, respectivamente, 65 e 44 vezes maior do que a obtida nas mesmas camadas do F1 (Tabela 2).

A competição de sulfato com nitrato por sítios de troca provavelmente explica a redução da adsorção de nitrato com o aumento do sulfato extraível. Nos latossolos de cerrado, as fontes de carga negativa são principalmente matéria orgânica e a caulinita

TABELA 3. Parâmetros da equação de Freundlich $\left(\mathbf{Y}=\mathbf{a} \mathbf{X}^{\mathbf{b}}\right)$ ajustada aos dados de nitrato adsorvido $(\mathrm{Y})$ e nitrato na solução $(\mathrm{X})$, nos diferentes sistemas de uso e profundidades do solo' ${ }^{1}$.

\begin{tabular}{lcll}
\hline Sistema & $\begin{array}{c}\text { Profundidade } \\
(\mathrm{cm})\end{array}$ & \multicolumn{1}{c}{$\mathrm{a}$} & $\mathrm{b}$ \\
\hline Cerrado & $0-20$ & $0,45(39,6)$ & $1,02(33,2)$ \\
& $20-40$ & $1,17(12,4)$ & $0,98(15,8)$ \\
& $40-80$ & $1,4(7,86)$ & $0,68(13,8)$ \\
Cerradão & $80-100$ & $2,26(5,0)$ & $0,60(11,2)$ \\
& $0-20$ & $0,45(12,3)$ & $0,92(11,3)$ \\
& $20-40$ & $0,95(18,5)$ & $0,94(22,3)$ \\
& $40-80$ & $1,2(7,4)$ & $0,78(10,9)$ \\
F1 & $80-100$ & $2,1(3,6)$ & $0,64(7,3)$ \\
& $0-20$ & $0,053(83,5)$ & $1,11(48,1)$ \\
& $20-40$ & $0,53(11,9)$ & $0,83(12,4)$ \\
& $40-80$ & $0,43(18,1)$ & $1,1(14,1)$ \\
F2 & $80-100$ & $0,91(36,4)$ & $1,2(36,3)$ \\
& $0-20$ & - & - \\
& $20-40$ & $0,22(28,2)$ & $0,62(35,3)$ \\
& $40-80$ & $0,24(21,3)$ & $1,16(13,6)$ \\
& $80-100$ & $1,2(12,9)$ & $0,86(16,9)$ \\
\hline Os valores entre parêntesis são os coeficientes de variação dos parâmetros \\
da equação.
\end{tabular}

(Silva \& Resck, 1997). Portanto, com a redução no teor de matéria orgânica em profundidade (Tabela 2), a carga líquida negativa é reduzida, diminuindo a repulsão do íon nitrato (Marcano-Martinez \& McBride, 1989; Motavalli et al., 1993). Motavalli et al. (1993), no mesmo solo sob cerradão, observaram que as cargas positivas aumentaram com a profundidade, enquanto as negativas diminuíram. MarcanoMartinez \& McBride (1989) sugerem que a menor capacidade de troca de ânions nos horizontes superficiais de dois latossolos do Distrito Federal devese ao bloqueamento químico dos sítios de carga positiva por ânions orgânicos.

A correlação entre o nitrato adsorvido e o $\Delta \mathrm{pH}$ só foi significativa $(\mathrm{r}=0,73 ; \mathrm{P}<0,01)$ quando se analisaram em conjunto apenas os solos do mesmo local, que apresentavam inversão de carga (cerrado, F1 e F2). Black \& Waring (1976) também obtiveram correlação significativa $(\mathrm{r}=0,87 ; \mathrm{P}<0,01)$ entre a quantidade de nitrato adsorvido e o $\Delta \mathrm{pH}$ em diferentes profundidades de um único solo, que apresentava, ao longo do perfil, mineralogia uniforme. No entanto, Black \& Waring (1979) verificaram que a correla-

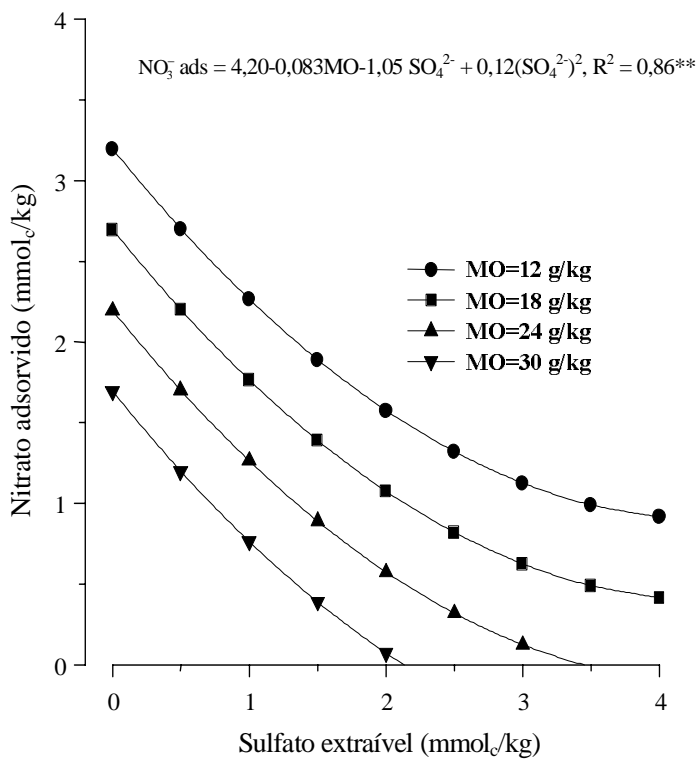

FIG. 2. Relação entre nitrato adsorvido, sulfato extraível e matéria orgânica das amostras de solos de várias profundidades.

Pesq. agropec. bras., Brasília, v.35, n.6, p.1199-1205, jun. 2000 
ção entre adsorção de nitrato e o $\Delta \mathrm{pH}$ era menor quando incluíram nos seus estudos solos com diferentes mineralogias. Neste estudo, os autores verificaram que as propriedades do solo que mais explicavam o aumento da adsorção de nitrato foram a superfície específica, $\mathrm{MO}$ e $\mathrm{pH}$ em $\mathrm{H}_{2} \mathrm{O}$. A diferença de mineralogia entre os dois solos, e conseqüentemente, superfícies específicas diferentes, podem explicar a falta de correlação entre essas duas características quando os dois solos foram analisados em conjunto. Provavelmente, a relação proporcional entre cargas positivas e negativas é o melhor indicador da maior ou menor adsorção de nitrato pelo solo.

$\mathrm{O}$ uso de gesso agrícola nos latossolos da região dos Cerrados pode aumentar a lixiviação de nitrato, mas a melhor distribuição do sistema radicular no perfil do solo, proporcionada pela lixiviação de $\mathrm{Ca}$, pode contrabalançar o aumento do potencial de perdas de nitrato. Sousa et al. (1992), no mesmo solo do sistema cerradão, observaram que as quantidades de nitrato no perfil do solo $(0-75 \mathrm{~cm})$ para os tratamentos sem aplicação de gesso e $6 \mathrm{t} / \mathrm{ha}$ deste insumo eram, respectivamente, 67 e $27 \mathrm{~kg} / \mathrm{ha}$ de nitrato. No entanto, a absorção de $\mathrm{N}$ pelo trigo foi de $30 \mathrm{~kg} / \mathrm{ha}$ a mais no tratamento que recebeu $6 \mathrm{t} / \mathrm{ha}$ de gesso. Esses autores observaram o mesmo comportamento nas culturas de milho e leucena.

A maior capacidade de adsorção de nitrato na subsuperfície desses solos estudados, independentemente de apresentarem inversão de carga ou não, pode contribuir para diminuir as perdas deste íon por lixiviação, o que poderia aumentar a eficiência das adubações nitrogenadas e reduzir o potencial de contaminação dos lençóis freáticos.

\section{CONCLUSÕES}

1. A adsorção de nitrato é maior na subsuperfície, independentemente do fenômeno de inversão de cargas elétricas do solo.

$2 \mathrm{O} \Delta \mathrm{pH}$ não é um bom índice para prever a adsorção de nitrato em solos com mineralogia distinta.
3. A maior adsorção de nitrato na subsuperfície dos dois latossolos está associada com os menores teores de matéria orgânica em profundidade.

4. A aplicação de gesso agrícola no solo reduz a adsorção de nitrato em subsuperfície do solo.

\section{REFERÊNCIAS}

BARROW, N.J. Studies on extraction and on availability to plant of adsorbed plus soluble sulphate. Soil Science, Baltimore, v.104, p.242-249, 1967.

BLACK, A.S.; WARING, S.A. Adsorption of nitrate, chloride and sulphate by some Highly Weathered soils from South-East Queensland. Australian Journal of Soil Research, Melbourne, v.17, n.2, p.271-282, 1979.

BLACK, A.S.; WARING, S.A. Nitrate leaching and adsorption in a Krasnozen from Redland Bay, Qld. I. Leaching of banded ammonium nitrate in a horticultural rotation. Australian Journal of Soil Research, Melbourne, v.14, n.2, p.171-180, 1976.

BOHN, H.L.; McNEAL, B.L.; O’CONNOR, G.A. Soil chemistry. New York: Wiley-Interscience, 1979. $329 \mathrm{p}$

BOWEN, W.T.; JONES, R.J.; CARSKY, R.J.; QUINTANA, J.O. Evaluation of the nitrogen submodel of CERES-maize following legume green manure incorporation. Agronomy Journal, Madison, v.85, n.1, p.153-158, 1993.

CAHN, M.D.; BOULDIN, D.R.; CRAVO, M.S. Nitrate sorption in the profile of an acid soil. Plant and Soil, Dordrecht, v.143, p.179-183, 1992.

DEPARTAMENTO NACIONAL DA PRODUÇÃO MINERAL. Projeto RADAMBRASIL. Folha SD.22 Goiás: geologia, geomorfologia, pedologia, vegetação, uso potencial da terra. Rio de Janeiro, 1983. 768p. (Levantamento de Recursos Naturais, 25)

EMBRAPA. Centro Nacional de Pesquisa de Solos (Rio de Janeiro, RJ). Manual de métodos de análise de solo. 2.ed. Rio de Janeiro, 1997. 212p. (EmbrapaCNPS. Documento, 1)

EMBRAPA. Serviço Nacional de Levantamento e Conservação de Solos (Rio de Janeiro, RJ). Levantamento de reconhecimento dos solos do Distrito 
Federal. Rio de Janeiro, 1978. 455p. (EmbrapaSNLCS. Boletim Técnico, 53).

KINJO, T.; KIEHL, E.J.; PRATT, P.F. Movimento do nitrato em colunas de terra de um Latossolo. Revista Brasileira de Ciência do Solo, Campinas, v.2, n.2, p.106-109, 1978.

KINJO, T.; PRATT, P.F. Nitrate adsorption: II. In competition with chloride, sulphate, and phosphate Soil Science Society of America. Proceedings, Madison, v.35, p.725-728, 1971

LINS, I.D.G.; COX, F.R. Effect of extractant and selected soil properties on predicting the optimum phosphorus fertilizer rate for growing soybeans under field conditions. Communications in Soil Science and Plant Analysis, New York, v.20, p.319-333, 1989.

MACEDO, J.; BRYANT, R.B. Morphology, mineralogy, and genesis of a hydrosequence of oxisols in Brazil Soil Science Society of America. Journal, Madison, v.51, p.690-698, 1987.

MARCANO-MARTINEZ, E.; McBRIDE, M.B Comparison of the titration and ion adsorption methods for surface charge measurement in oxisols. Soil Science Society of America. Journal Madison, v.53, p.1040-1045, 1989.

MOTAVALLI, P.P.; DUXBURY, J.M.; SOUSA, D.M.G de. The influence of organic soil amendments on sulphate adsorption and sulfur availability in a Brazilian oxisol. Plant and Soil, Dordrecht, v.154 p.301-308, 1993.
NEWBOULD, P. The use of nitrogen fertilizer in agriculture: where do we go practically and ecologically? Plant and Soil, Dordrecht, v.115, p.297-311, 1989.

PARFITT, R.L. Chemical properties of variable charge soils. In: THENG, B.K.G. Soils with variable charge. Palmerston North : New Zealand Society of Soil Science, 1980. p.167-194.

RAIJ, B. van; CAMARGO, O.A. Nitrate elution from soil columns of three Oxisols and one Alfisol. In INTERNATIONAL CONGRESS OF SOIL SCIENCE, 10., 1974, Moscow. Transactions. Moscow : International Society of Soil Science, 1974. v. 2 , p. 384-391

SAS INSTITUTE (Cary, Estados Unidos). SAS/STAT user's guide: version 6. 4.ed. Cary, 1989. v.2.

SILVA, J.E.; RESCK, D.V.S. Matéria orgânica do solo. In: VARGAS, M.A.T.; HUNGRIA, M. (Ed.). Biologia dos solos dos Cerrados. Planaltina, DF : Embrapa-CPAC, 1997. p.467-524

SOUSA, D.M.G. de; LOBATO, E.; RITCHEY, K.D.; REIN, T.A. Resposta de culturas anuais e leucena a gesso no Cerrado. In: SEMINÁRIO SOBRE O USO DO GESSO NA AGRICULTURA, 2., 1992, Uberaba. Anais. [São Paulo] : Ibrafos, 1992. p.275306.

SUHET, A.R.; PERES, J.R.R; VARGAS, M.A.T. Nitrogênio. In: GOEDERT, W.J. (Ed.). Solos dos Cerrados: tecnologias e estratégias de manejo. São Paulo: Nobel, 1986. p.167-202 